\title{
SINGULARITIES OF RICHARDSON VARIETIES
}

\author{
Allen Knutson, Alexander Woo and Alexander Yong
}

\begin{abstract}
This paper gives a short proof that essentially all questions concerning singularities of Richardson varieties reduce to corresponding questions about Schubert varieties. Consequently, one quickly deduces new and previously known results.
\end{abstract}

\section{Introduction and the main result}

Let $G$ denote a simple reductive linear algebraic group over an algebraically closed field $\mathbb{k}$. Fix a choice of Borel and opposite Borel subgroups $B$ and $B_{-}$, and let $T=B \cap B_{-}$ be the associated maximal torus. A generalized flag variety is a variety $G / P$, where $P \supseteq B$ is a parabolic subgroup. A $B$-orbit closure $X_{w}:=\overline{B w P / P} \subseteq G / P$ is known as a Schubert variety. Here $w$ is an element of the Weyl group $W \cong N(T) / T$ of $G$. If we let $W_{P}$ be the parabolic subgroup of $W$ associated to $P$, the choice of a different coset representative in $w W_{P}$ gives the same Schubert variety.

There has been significant interest in invariants of singularities of Schubert varieties; two surveys include [BilLak01, Bri05]. More generally, one wishes to similarly understand singularities of other special subvarieties $X$ of various $G / P$. Two examples of such subvarieties $X$ are $K$-orbit closures (where $K$ is a symmetric subgroup of $G$ ) and Peterson varieties. Combinatorics important to the study of Schubert varieties shows up in these cases; for example, permutation pattern avoidance appears in both the study of $K$-orbits [McgTra09] and Peterson varieties [InsYon12].

This paper concerns Richardson varieties, which are the varieties $X_{w}^{v}:=X_{w} \cap$ $X^{v}$, where $X^{v}=\overline{B_{-} v P / P}$ is an opposite Schubert variety. When $v \leq w$ in Bruhat order, $X_{w}^{v}$ is nonempty, reduced and irreducible [Ric92]. Richardson varieties play a prominent role in Schubert calculus, as their classes in the cohomology ring of $G / P$ satisfy $\left[X_{w}^{v}\right]=\left[X_{w}\right] \cdot\left[X_{w_{o} v}\right]$. Hence one can study the product of Schubert classes and more specifically the generalized Littlewood-Richardson coefficients $C_{v, u}^{w}$ defined by $\left[X_{u}\right] \cdot\left[X_{v}\right]=\sum_{w} C_{v, u}^{w}\left[X_{w}\right]$ by studying Richardson varieties. Several recent papers have studied the singularities of Richardson varieties using various techniques, including standard monomial theory [KreLak04], intersection theory [BilCos12, Bal12], and Frobenius splitting [KnuLamSpe09]. Indeed, [BilCos12, KnuLamSpe09] study a generalization known as the projected Richardson varieties.

Our main result states that, given any point $x \in X_{w}^{v}$, the local properties of $X_{w}^{v}$ at $x$ are completely determined by the Schubert cell $X_{\sigma}^{\circ}$ and opposite Schubert cell $X_{\circ}^{\tau}$ to which $x$ belongs. Furthermore, for any invariant or property whose behavior under

Received by the editors December 31, 2012.

2000 Mathematics Subject Classification. 14M15, 14N15.

Key words and phrases. Richardson varieties, Schubert varieties, singularities. 
product of varieties is understood, its behavior on $X_{w}^{v}$ at $x$ can be calculated from its behavior on $X_{w}$ at points of $X_{\circ}^{\tau}$ and its behavior on $X^{v}$ at points of $X_{\sigma}^{\circ}$. More precisely:

Theorem 1.1. Let $\mathcal{P}$ be a local invariant of varieties that is preserved under products with affine space. Suppose there exists a function $f_{\mathcal{P}}$ such that, for any varieties $X$ and $Y$,

$$
\mathcal{P}(x \times y, X \times Y)=f_{\mathcal{P}}(\mathcal{P}(x, X), \mathcal{P}(y, Y)), \quad \text { for any } x \in X, y \in Y .
$$

Let $x P \in X_{w}^{v}$, and suppose $x P \in(B \sigma P / P) \cap\left(B_{-} \tau P / P\right)$ for some $\sigma, \tau \in W$. Then

$$
\mathcal{P}\left(x P, X_{w}^{v}\right)=f_{\mathcal{P}}\left(\mathcal{P}\left(\sigma P, X_{w}\right), \mathcal{P}\left(\tau P, X^{v}\right)\right)=f_{\mathcal{P}}\left(\mathcal{P}\left(x P, X_{w}\right), \mathcal{P}\left(x P, X^{v}\right)\right) .
$$

Our proof gives a local isomorphism, up to a product with affine space, between a neighborhood of a point $x P \in X_{w}^{v}$ and a product of local charts on $X_{w}$ and $X^{v}$. This isomorphism is $T$-equivariant. Furthermore, the reader can see from the proof that our theorem applies not only to ( $T$-equivariant) intrinsic invariants, but also to local invariants that are relative to the embedding of $X_{w}^{v}$ in $G / P$ (for example local $T$-equivariant cohomology classes).

We emphasize that in Theorem 1.1, $x P$ need not be a $T$-fixed point. For Schubert varieties, the elements of $B$ provide local isomorphisms between any point $x P \in X_{w}$ and a $T$-fixed point; thus one can assume $x P \in(G / P)^{T}$ if one is interested in local properties. However, in general the largest subgroup of $G$ that fixes $X_{w}^{v}$ is $T$, which is not large enough to put every point in the orbit of a $T$-fixed point.

Call an invariant $\mathcal{P}$ factorizable with respect to $f_{\mathcal{P}}$ if it satisfies (1.1). Almost all interesting local invariants are factorizable. We now mention three important previously studied special cases of our theorem.

Example 1. $\mathcal{P}=$ "is smooth" is factorizable with respect to $f_{\mathcal{P}}=$ "logical and".

Let $\operatorname{Singlocus}(X)$ denote the singular locus of a variety $X$. The following was first stated in [BilCos12, Corollary 2.9]. Their proof invokes Kleiman's transversality theorem; it is also immediate from Theorem 1.1 combined with Example 1:

Corollary 1.2. $\operatorname{Singlocus}\left(X_{w}^{v}\right)=\left(\operatorname{Singlocus}\left(X_{w}\right) \cap X^{v}\right) \cup\left(X_{w} \cap \operatorname{Singlocus}\left(X^{v}\right)\right)$.

Example 2. $\mathcal{P}=$ "is normal and Cohen-Macaulay with rational singularities" is also factorizable with respect to $f_{\mathcal{P}}=$ "logical and".

It is well known that every Schubert variety is normal and Cohen-Macaulay with rational singularities; see [Bri05] for proofs and historical remarks. The following was originally proved in [Bri02] in characteristic zero and [KnuLamSpe09, Appendix A] in general. Their proofs use a generalization of the Bott-Samelson resolution and, in the latter case, the Frobenius splitting of $G / P$.

Corollary 1.3. The Richardson variety $X_{w}^{v}$ is Cohen-Macaulay and normal with rational singularities.

Example 3. Consider the $\mathbb{Z}$-graded Hilbert series of $\operatorname{gr}_{\mathfrak{m}_{p}} \mathcal{O}_{p, Z}$, the associated graded ring of the local ring $\mathcal{O}_{p, Z}$, which is denoted $\operatorname{Hilb}\left(\operatorname{gr}_{\mathfrak{m}_{p}} \mathcal{O}_{p, Z}, q\right)$. The $H$-polynomial $H_{p, Z}(q)$ is defined by

$$
\operatorname{Hilb}\left(\operatorname{gr}_{\mathfrak{m}_{p}} \mathcal{O}_{p, Z}, q\right)=\frac{H_{p, Z}(q)}{(1-q)^{\operatorname{dim} Z}}
$$


and $H_{p, Z}(1)$ is the Hilbert-Samuel multiplicity mult $p, Z$. Let $\mathcal{P}$ be either $H_{p, Z}(q)$ or mult $_{p, Z}$, and $f_{\mathcal{P}}=$ "(usual) multiplication". Since "taking associated graded commutes with tensor product," $\mathcal{P}$ is factorizable with respect to $f_{\mathcal{P}}$.

Corollary 1.4. Let $x P \in X_{w}^{v}$. Then $H_{x P, X_{w}^{v}}(q)=H_{x P, X_{w}}(q) \cdot H_{x P, X^{v}}(q)$.

In [LiYon11], the $H$-polynomial was studied for Schubert varieties, where it was conjectured $H_{v P, X_{w}}(q) \in \mathbb{N}[q]$. Corollary 1.4 extends the conjecture to Richardson varieties.

The consequence that

$$
\operatorname{mult}_{x P, X_{w}^{v}}=\operatorname{mult}_{x P, X_{w}} \cdot \operatorname{mult}_{x P, X}
$$

also appears to be new. Previously, (1.2) was proved for minuscule $G / P$ in [KreLak04, Remark 7.6.6] when $x P \in(G / P)^{T}$ (which is the case $\sigma=\tau$ ).

This result was generalized by M. Balan [Bal12] who proved (1.2) for all points $x P$, again assuming that $P$ is minuscule. (A different generalization in the minuscule setting appears in [BilCos12, Remark 2.15].)

In Section 2, we prove Theorem 1.1. Our proof is elementary, at least if one accepts standard algebraic groups language. It uses a variation of [KazLus79, Lemma A.4], that relies on a variation on standard results about unipotent groups found, for example, in [Hum75, Section 28.1]. We also include a variant of the proof that avoids the language of algebraic groups (valid only in type $A$, but otherwise essentially the same as the general proof) in Section 3. In that section, we also discuss some further consequences of Theorem 1.1.

\section{Proof of Theorem 1.1}

The projection map $\rho: G / B \rightarrow G / P$ is a fibration with fibers locally isomorphic to affine space. Thus, since our local invariant is constant under product with affine space, its value at a point $x P \in \overline{B w P / P} \cap \overline{B_{-} v P / P}$ is the same as that for any point in $\rho^{-1}(x P) \subseteq \overline{B w^{P} B / B} \cap \overline{B v_{P} B / B}$, where $w^{P}$ is the maximal length coset representative of $w \in W / W_{P}$ and $v_{P}$ is the minimal length coset representative of $v \in W / W_{P}$. Therefore, it follows that to check correctness of Theorem 1.1, we can assume $P=B$.

Let $X_{u}^{\circ}=B u B / B$ and $X_{\circ}^{u}=B_{-} u B / B$ be the Schubert and opposite Schubert cells for $u$. The proof depends on the following version of [KazLus79, Lemma A.4].

Lemma 2.1. Given any $u \in W$, there exists an isomorphism

$$
\eta=\left(\eta_{1}, \eta_{2}\right): u X_{\circ}^{\mathrm{id}} \rightarrow X_{\circ}^{u} \times X_{u}^{\circ}
$$

such that, for any $x \in u X_{\circ}^{\mathrm{id}}, \eta_{1}(x)$ and $x$ are in the same Schubert cell, and $\eta_{2}(x)$ and $x$ are in the same opposite Schubert cell.

The result [KazLus79, Lemma A.4] states that there is such a T-equivariant isomorphism but does not state the additional properties about it asserted in Lemma 2.1.

Our proof of Lemma 2.1 depends on the following lemma about algebraic groups.

Lemma 2.2. Let $U$ be a unipotent group on which $T$ acts without nontrivial fixed points, while $\mathfrak{u}=\operatorname{Lie}(U)$ is the direct sum of one-dimensional eigenspaces for $T$ 
corresponding to characters $\alpha$ with distinct connected kernels $T_{\alpha}$. Furthermore, let $U_{1}$ and $U_{2}$ be $T$-stable subgroups of $U$ such that $U_{1} \cap U_{2}=\{\mathrm{id}\}, U_{1} U_{2}=U$, and the corresponding Lie algebras satisfy $\mathfrak{u}=\mathfrak{u}_{1} \oplus \mathfrak{u}_{2}$. Then there exists an isomorphism of varieties

$$
\sigma: U \rightarrow U_{1} \times U_{2}
$$

such that the factors $\sigma_{1}$ and $\sigma_{2}$ satisfy $\sigma_{1}(u) \in U_{2} u$ and $\sigma_{2}(u) \in U_{1} u$.

Note that $\sigma$ will not be a group homomorphism unless $U_{1}$ and $U_{2}$ commute.

Proof. It is a standard fact (see, for example, [Hum75, Section 28.1]) that the product morphisms $\pi: U_{1} \times U_{2} \rightarrow U$ and $\kappa: U_{1} \times U_{2} \rightarrow U$ given by $\pi\left(u_{1}, u_{2}\right)=u_{1} u_{2}$ and $\kappa\left(u_{1}, u_{2}\right)=u_{2} u_{1}$ are bijective morphisms of varieties. The hypothesis on the Lie algebras implies they are actually isomorphisms (of varieties) since the differential is everywhere injective; see the local isomorphism criterion, for example, in [Har92, Theorem 14.9]. However, the inverses of these two maps each only satisfy one of our two requirements that $\sigma_{1}(u) \in U_{2} u$ and $\sigma_{2}(u) \in U_{1} u$.

Let $\pi_{2}^{-1}$ and $\kappa_{1}^{-1}$ be respectively the inverses of $\pi$ and $\kappa$ followed by projection onto respectively the second and first factors. We now have a map $\sigma=\kappa_{1}^{-1} \times \pi_{2}^{-1}$ : $U \rightarrow U_{1} \times U_{2}$. It remains to prove that $\kappa_{1}^{-1} \times \pi_{2}^{-1}$ is an isomorphism (of schemes), as once that is done, $\sigma$ is our desired map, as follows. Since $\sigma_{1}=\kappa_{1}^{-1}$, so $\sigma_{1}(u) \in U_{2} u$, and since $\sigma_{2}=\pi_{2}^{-1}$, so $\sigma_{2}(u) \in U_{1} u$.

We prove $\kappa_{1}^{-1} \times \pi_{2}^{-1}$ is an isomorphism by induction on the dimension of $U$. Since $U$ is unipotent, it is nilpotent and thus has a nontrivial center $U^{\prime}$ [Hum75, Lemma 17.4(c)]. This center is clearly $T$-stable. Therefore $U_{1}^{\prime}=U_{1} \cap U^{\prime}$ and $U_{2}^{\prime}=U_{2} \cap U^{\prime}$ are $T$-stable subgroups of $U^{\prime}$.

As $U^{\prime}, U_{1}^{\prime}$ and $U_{2}^{\prime}$ are unipotent and $T$-stable, they are spanned by products of root subgroups $U_{\alpha}$ [Hum75, Proposition 28.1]. These root subgroups are a subset of those spanning $U, U_{1}$ and $U_{2}$ respectively. Hence, it follows $U_{1}^{\prime} U_{2}^{\prime}=U^{\prime}$. Therefore, $\pi$ and $\kappa$ restrict to isomorphisms $\tilde{\pi}, \widetilde{\kappa}: U_{1}^{\prime} \times U_{2}^{\prime} \rightarrow U^{\prime}$. Since $U_{1}^{\prime}$ and $U_{2}^{\prime}$ commute, $\widetilde{\kappa}_{1}^{-1} \times \widetilde{\pi}_{2}^{-1}$ is actually the same as both $\widetilde{\kappa}^{-1}$ and $\widetilde{\pi}^{-1}$ on $U^{\prime}$ and hence invertible.

Furthermore, because $U^{\prime}$ is central, $\pi$ and $\kappa$ induce maps $\bar{\pi}, \bar{\kappa}: U_{1} / U_{1}^{\prime} \times U_{2} / U_{2}^{\prime} \rightarrow$ $U / U^{\prime}$.

(It is straightforward to check the maps are well defined.) By induction, the map

$$
\bar{\kappa}_{1}^{-1} \times \bar{\pi}_{2}^{-1}: U / U^{\prime} \rightarrow U_{1} / U_{1}^{\prime} \times U_{2} / U_{2}^{\prime}
$$

is an isomorphism. (Technically, the induction applies to the natural embeddings, which are isomorphisms onto their images, of $U_{1} / U_{1}^{\prime}$ and $U_{2} / U_{2}^{\prime}$ in $U / U^{\prime}$.)

Summarizing, we have maps in a commutative diagram:

$$
\begin{aligned}
& 0 \longrightarrow U^{\prime} \longrightarrow U \longrightarrow U / U^{\prime} \longrightarrow 0 \\
& \downarrow \widetilde{\kappa}_{1}^{-1} \times \widetilde{\pi}_{2}^{-1} \quad \downarrow \sigma=\kappa_{1}^{-1} \times \pi_{2}^{-1} \quad \downarrow \bar{\kappa}_{1}^{-1} \times \bar{\pi}_{2}^{-1} \\
& 0 \longrightarrow U_{1}^{\prime} \times U_{2}^{\prime} \longrightarrow U_{1} \times U_{2} \longrightarrow U_{1} / U_{1}^{\prime} \times U_{2} / U_{2}^{\prime} \longrightarrow 0
\end{aligned}
$$

It is easy to check from the definitions that $\sigma$ not only restricts to a group homomorphism on $U^{\prime}$ but moreover that $\sigma(a b)=\sigma(a) \sigma(b)$ whenever $a$ or $b$ is in $U^{\prime}$. Using this, a straightforward diagram chase similar to the proof of the five-lemma implies $\sigma$ is a bijection.

By our hypotheses, the equality of tangent spaces $T_{p}(U)=\mathfrak{u}=\mathfrak{u}_{1} \oplus \mathfrak{u}_{2}=T_{(a, b)}$ $\left(U_{1} \times U_{2}\right)$ holds when $p, a, b$ are the identity. Hence, $d \sigma$ is an injection at the identity. 
However, injectivity holds everywhere else since $\sigma$ is $T$-equivariant, the identity is the only $T$-fixed point, and failure for the differential to be injective is a closed condition. Therefore, $\sigma$ is an isomorphism of schemes, by another use of the local criterion for isomorphism.

Proof of Lemma 2.1: Let $U_{+} \subset B$ and $U_{-} \subset B_{-}$respectively denote the unipotent subgroups of the Borel and opposite Borel subgroups of $G$. Given $u \in W$, we use Lemma 2.2 in the case where $U=u U_{-} u^{-1}, U_{1}=U_{-} \cap u U_{-} u^{-1}$, and $U_{2}=U_{+} \cap$ $u U_{-} u^{-1}$. Lemma 2.2 gives us an isomorphism

$$
\sigma: U \rightarrow U_{1} \times U_{2}
$$

such that $\sigma_{1}(u)$ and $u$ differ by left multiplication by an element of $U_{2}$ and $\sigma_{2}(u)$ and $u$ differ by left multiplication by an element of $U_{1}$.

Now note that we have isomorphisms $m_{0}: U \rightarrow u X_{\circ}^{\mathrm{id}}, m_{1}: U_{1} \rightarrow X_{\circ}^{u}$, and $m_{2}: U_{2} \rightarrow X_{u}^{\circ}$ each defined by $m_{i}(g)=g u B$. Let $\eta=\left(m_{1} \times m_{2}\right) \circ \sigma \circ m_{0}^{-1}$. Since by Lemma 2.2, $\sigma_{1}(g) \in U_{2} g$ for any $g \in U$ and $U_{2} \subseteq U_{+} \subset B, \eta_{1}(g u B) \in B g u B$ and hence this point is in the same Schubert cell as $g u B$. Similarly since $U_{1} \subset B_{-}$, the points $\eta_{2}(g u B)$ and $g u B$ are in the same opposite Schubert cell.

Proof of Theorem 1.1: Let $x B \in G / B$. Since the charts $\left\{u X_{\circ}^{\mathrm{id}}\right\}_{u \in W}$ cover $G / B$, we can fix $u \in W$ such that $x B \in u X_{\circ}^{\text {id }}$. Also let $\eta$ be the map from Lemma 2.1.

We have the Bruhat and opposite Bruhat decompositions

$$
X_{w}=\coprod_{\sigma \leq w} X_{\sigma}^{\circ} \text { and } X^{v}=\coprod_{\tau \geq v} X_{\circ}^{\tau} .
$$

Therefore by (2.1), $x B \in X_{w}^{v}$ if and only if $x B \in X_{\sigma}^{\circ}$ for some fixed $\sigma \leq w$ and $x B \in X_{\circ}^{\tau}$ for some fixed $\tau \geq v$. By Lemma 2.1 we know $\eta_{1}(x B)$ and $x B$ are in the same Schubert cell, and we also know $\eta_{2}(x B)$ and $x B$ are in the same opposite Schubert cell. Therefore, $x B \in X_{w}^{v}$ if and only if $\eta_{1}(x B) \in X_{w}$ and $\eta_{2}(x B) \in X^{v}$.

Therefore, $\eta$ restricts to an isomorphism

$$
\left.\eta\right|_{X_{w}^{v}}: u X_{\circ}^{\mathrm{id}} \cap X_{w}^{v} \rightarrow\left(X_{\circ}^{u} \cap X_{w}\right) \times\left(X_{u}^{\circ} \cap X^{v}\right) .
$$

This isomorphism is scheme-theoretic since both sides are reduced. (The right-hand side is reduced since it is a product of two integral schemes over their base field and thus integral.)

Now suppose $x B \in X_{w}^{v}$. Since $\mathcal{P}$ is a local invariant,

$$
\mathcal{P}\left(x B, X_{w}^{v}\right)=\mathcal{P}\left(x B, u X_{\circ}^{\mathrm{id}} \cap X_{w}^{v}\right) .
$$

Since $\eta$ is an isomorphism,

$$
\mathcal{P}\left(x B, u X_{\circ}^{\mathrm{id}} \cap X_{w}^{v}\right)=\mathcal{P}\left(\eta(x B),\left(X_{\circ}^{u} \cap X_{w}\right) \times\left(X_{u}^{\circ} \cap X^{v}\right)\right) .
$$

By the hypothesis that $\mathcal{P}$ factorizes with respect to $f_{\mathcal{P}}$,

$\mathcal{P}\left(\eta(x B),\left(X_{\circ}^{u} \cap X_{w}\right) \times\left(X_{u}^{\circ} \cap X^{v}\right)\right)=f_{\mathcal{P}}\left(\mathcal{P}\left(\eta_{1}(x B), X_{\circ}^{u} \cap X_{w}\right), \mathcal{P}\left(\eta_{2}(x B), X_{u}^{\circ} \cap X^{v}\right)\right)$.

If $x B \in X_{\sigma}^{\circ} \cap X_{\circ}^{\tau}$, then by Lemma 2.1 we know $\eta_{1}(x B) \in X_{\sigma}^{\circ}$ and $\eta_{2}(x B) \in X_{\circ}^{\tau}$.

Since in a Schubert variety every point is locally isomorphic (by the action of $B$ ) to the $T$-fixed point in its Schubert cell, we have:

$$
\mathcal{P}\left(\eta_{1}(x B), X_{\circ}^{u} \cap X_{w}\right)=\mathcal{P}\left(\eta_{1}(x B), X_{w}\right)=\mathcal{P}\left(\sigma B, X_{w}\right) .
$$


The first equality uses [KazLus79, Lemma A.4] (of which we have just proved a stronger version), which states that $\left(X_{\circ}^{u} \cap X_{w}\right) \times \mathbb{k}^{\ell(u)} \cong u X_{\circ}^{\text {id }} \cap X_{w}$. We are also using the assumption that $\mathcal{P}$ is invariant under Cartesian product with affine space.

The same arguments apply to the opposite Schubert varieties (using the action of $\left.B_{-}\right)$; hence:

$$
\mathcal{P}\left(\eta_{2}(x B), X_{u}^{\circ} \cap X^{v}\right)=\mathcal{P}\left(\eta_{2}(x B), X^{v}\right)=\mathcal{P}\left(\tau B, X^{v}\right) .
$$

Combining the above we obtain

$$
\mathcal{P}\left(x B, X_{w}^{v}\right)=f_{\mathcal{P}}\left(\mathcal{P}\left(\sigma B, X_{w}\right), \mathcal{P}\left(\tau B, X^{v}\right)\right),
$$

as desired.

\section{Further consequences and comments}

3.1. Other singularity invariants. For brevity, we refer the reader to [WooYon08] for discussion of properties of Schubert varieties of interest to us. The purpose of this section is to explain the extension of this discussion to Richardson varieties.

There are two kinds of invariants $\mathcal{P}$ we are interested in. The first is of the "yes/no" kind. As in Corollary 1.2, they are factorizable by $f_{\mathcal{P}}=$ "logical and". If non $\mathcal{P} \operatorname{locus}(X)$ is the set of points in $X$ where $\mathcal{P}$ takes on the value "no", then we have

$$
\operatorname{non} \mathcal{P} \operatorname{locus}\left(X_{w}^{v}\right)=\left(\operatorname{non} \mathcal{P} \operatorname{locus}\left(X_{w}\right) \cap X^{v}\right) \cup\left(X_{w} \cap \operatorname{non} \mathcal{P} \operatorname{locus}\left(X^{v}\right)\right) .
$$

In [WooYon06] it was determined which Schubert varieties are Gorenstein. The property $\mathcal{P}=$ "Gorenstein" is factorizable with respect to $f_{\mathcal{P}}=$ "logical and" since being Gorenstein is a homological property (and the total complex of the double complex formed by tensoring two free resolutions is exact in this case). Thus, we record:

Corollary 3.1. (3.1) holds for $\mathcal{P}=$ "is Gorenstein".

In [WooYon06, WooYon08] a conjectural description was given of the nonGorenstein locus of any Schubert variety. This conjecture was stated using interval pattern avoidance in the case of $G L_{n} / B$; furthermore, the conjecture was made explicit and proved for minuscule $G / P$ by N. Perrin [Per09]. In such cases, one can combine these combinatorial descriptions with Corollary 3.1 to obtain descriptions of non-Gorlocus $\left(X_{w}^{v}\right)$. Recently, Darayon [Dar12] determined which Richardson varieties in Grassmannians are arithmetically Gorenstein under the standard embedding. (Theorem 1.1 does not apply to the cone singularity.)

Unfortunately, even Corollary 3.1 combined with the results in [WooYon06] does not provide a characterization for when $X_{w}^{v} \subseteq G L_{n} / B$ is Gorenstein. One seems to need a (compatible) characterization for the non-Gorenstein locus of a Schubert variety in general.

A stronger property than Gorensteinness is that of $\mathcal{P}=$ "is a local complete intersection (lci)". This $\mathcal{P}$ factorizes with respect to $f_{\mathcal{P}}=$ "logical and", and thus (3.1) again applies. Recently, a characterization of which $X_{w} \subseteq G L_{n} / B$ are local complete intersections has been determined by H. Úlfarsson and the second author [UlfWoo11]. To further determine when $X_{w}^{v}$ is a local complete intersection, one also seems to need a characterization of the lci locus of a Schubert variety. 
The second type of invariant we are interested in takes values in $\mathbb{Z}$ or some (Laurent) polynomial ring. Suppose $\mathcal{P}$ is factorizable with respect to $f_{\mathcal{P}}=$ "usual multiplication of numbers or (Laurent) polynomials". If we let $\mathcal{P}_{x, X}$ denote the value taken at $x \in X$, then just as in Corollary 1.4, we have

$$
\mathcal{P}_{x P, X_{w}^{v}}=\mathcal{P}_{x P, X_{w}} \cdot \mathcal{P}_{x P, X^{v}}
$$

for any $x P \in X_{w}^{v}$.

More refined than knowing if a point $x \in X$ is Gorenstein is to know the CohenMacaulay type of that point. Let $\mathcal{P}_{x, X}=C M_{x, X} \in \mathbb{N}$ denote this statistic. (When $C M_{x, X}=1$ then $x$ is a Gorenstein point, assuming $X$ is Cohen-Macaulay, which is always true in our situation.) Again, since $\mathcal{P}$ is a homological property, it factorizes with respect to $f_{\mathcal{P}}=$ "(usual multiplication)". Thus, we obtain another example of (3.2):

Corollary 3.2. (3.2) holds for $\mathcal{P}=$ "Cohen-Macaulay type".

Let $P_{v, w}(q) \in \mathbb{N}[q]$ denote a Kazhdan-Lusztig polynomial. Owing to its interpretation as a Poincaré polynomial for local intersection cohomology at $v B$ in $X_{w} \subseteq G / B$ (together with a Kunneth-type formula for local intersection cohomology), one can apply (3.2), i.e.,

$$
Q_{x, w, v}(q)=P_{\sigma, w}(q) \cdot P_{w_{0} \tau, w_{0} v}(q),
$$

where $Q_{x, w, v}(q)$ is the Poincaré polynomial for local intersection cohomology of $X_{w}^{v}$ at $x B$ and where $x B \in X_{\sigma}^{\circ}$ and $x B \in X_{\circ}^{\tau}$ (as in the Introduction section).

Work of $\mathrm{Li}$ and the second author [LiYon11] suggests an analogy between the polynomials $P_{v, w}(q)$ and $H_{v P, X_{w}}(q)$. In particular, for Grassmannians and covexillary Schubert varieties, it is shown that $P_{v, w}(q) \preceq H_{v P, X_{w}}(q)$, where $\preceq$ means "coefficientwise $\leq "$. This therefore lifts to Richardson varieties.

Wyser [Wys12] has recently proved that certain $K$-orbits (for example, certain $K=G L_{p} \times G L_{q}$ orbits in $\left.G L_{n} / B\right)$ are isomorphic to Richardson varieties. Therefore, our results apply to those $K$-orbits. Further discussion may appear elsewhere.

3.2. Another proof of Lemma 2.1, when in Type A. Assume $x$ is the generic matrix in $u X_{\circ}^{\mathrm{id}}$ : the rows are a generic matrix in $U_{-}$, but are $u$-permuted. Let $z_{i j}$ be the entry in row $i$ and column $j$. Note $u(i)=j$ means there is a 1 in column $i$ and row $j$ of the matrix. See Example 4.

Define $\eta_{1}(x)$ to be obtained by "sweeping up using the 1's in each column": take the column $j_{1}$ whose " 1 " (coming from $u$ ) is in row $n$ (so $j_{1}=u^{-1}(n)$ ) and do upward row operations, so that all entries in column $j_{1}$ strictly above row $n$ are 0 . Then find the column $j_{2}$ whose " 1 " is in row $n-1$ and do operations that place 0 above that " 1 " etc. Declare the result after sweeping in all $n$ columns to be $\eta_{1}(x)$. (The resulting matrix can be obtained by any sequence of row operations that put 0 's above each "1"; however the stated order is most efficient, as no column has to be swept more than once.) Clearly $\eta_{1}(x)$ is in $B u B / B$ and is in the same Schubert cell as $x$ since the former comes from the latter by left multiplication of a matrix from $B$.

Similarly, define $\eta_{2}(x) \in B_{-} u B / B$ by downward sweeping operations, starting with the column whose "1" (from $u$ ) appears in row 1 , etc. Likewise, $\eta_{2}(x)$ and $x$ are in the same opposite Schubert cell. 
Let

$$
\begin{aligned}
D^{\text {up }} & =\left\{(i, j) \in[n] \times[n]: i>\pi(j) \text { and } j<\pi^{-1}(i)\right\}, \\
D^{\text {down }} & =\left\{(i, j) \in[n] \times[n]: i<\pi(j) \text { and } j<\pi^{-1}(i)\right\} .
\end{aligned}
$$

These are the positions in $\eta_{1}(x)$ and $\eta_{2}(x)$ respectively not (a priori) equal to 0 or 1 .

Claim 3.3. (i) In $\eta_{1}(x)$, the entry in position $(i, j) \in D^{\text {up }}$ is an expression of the form $z_{i j}+f$ where $f$ is a polynomial in the generic entries $z_{a, b}$ where either $b=j$ and $a>i$, or $b>j$.

(ii) Similarly, in $\eta_{2}(x)$, the entry in position $(i, j) \in D^{\text {down }}$ is an expression of the form $z_{i j}+g$ where $g$ is a polynomial in the generic entries $z_{a, b}$ where either $b=j$ and $a<i$, or $b>j$.

Proof. The argument for (i) is the upside down version of (ii). Let us argue the latter case (where one does downward sweeping). The only 1's we use to sweep that affect some position $(i, j) \in D^{\text {down }}$ are those strictly northeast of $(i, j)$. Say this 1 is in position $\left(i^{\prime}, j^{\prime}\right.$ ) (with $i^{\prime}<i$ and $j^{\prime}>j$ ). Then the row operation involving this 1 converts the entry $y_{i, j}$ in position $(i, j)$ to $y_{i j}-y_{i^{\prime}, j} y_{i, j^{\prime}}$, and the result follows by induction on $j \leq n$.

We now recover all generic entries $z_{i j}$ in $x$ from the entries of $\eta_{1}(x)$ and $\eta_{2}(x)$. Inductively, we argue that, if we know all such entries in columns $k, k+1, \ldots, n$ of $x$, we can deduce the entries in column $k-1$ of $x$.

For the base case of the argument, note that column $n$ of $x$ consists of only 0's and 1's, so there is nothing to do. In the induction step, the argument depends on the relative positions of the 1's in columns $k-1$ and $k$ :

Case I - The 1 in column $k-1$ is higher (lower index) than the one in column $k$ : Suppose $u(k-1)=b$ and $u(k)=c$ where $b<c$. First consider positions $(q, k-1)$ for $q<b$. We use $\eta_{2}(x)$. Either position $(1, k-1)$ is 0 in $x$ (because there is a 1 to the left of column $k-1$ in the first row), or $z_{1, k-1}$ appears in that position of $\eta_{2}(x)$ and hence is determined. Then by Claim 3.3(ii) and induction, working top to bottom, we determine each $z_{q, k-1}$ for $q<b$.

Now consider indices $(r, k-1)$ for $r>b$. This time note position $(n, k-1)$ is either 0 in $x$ or $z_{n, k-1}$ appears there in both $x$ and $\eta_{1}(x)$ and hence is determined. Then the remaining generic variables of $x$ in column $k-1$ are obtained from $\eta_{1}(x)$ by working bottom to top, applying induction and Claim 3.3(i).

Case II - The 1 in column $k-1$ is lower (higher index) than the one in column $k$ : The argument is the upside down version of Case I, reversing the roles of $\eta_{1}(x)$ and $\eta_{2}(x)$ in the obvious way.

Our second proof of Theorem 1.1 (in type $A$ ) now concludes as in Section 2.

Example 4. Let $u=31542 \in S_{5}$. A generic matrix in $u X_{\circ}^{\mathrm{id}}$ has a standard form

$$
x=\left(\begin{array}{ccccc}
z_{11} & 1 & 0 & 0 & 0 \\
z_{21} & z_{22} & z_{23} & z_{24} & 1 \\
1 & 0 & 0 & 0 & 0 \\
z_{41} & z_{42} & z_{43} & 1 & 0 \\
z_{51} & z_{52} & 1 & 0 & 0
\end{array}\right)
$$


Then

$$
\eta_{1}(x)=\left(\begin{array}{ccccc}
0 & 1 & 0 & 0 & 0 \\
0 & z_{22}-z_{24}\left(z_{42}-z_{52} z_{43}\right)-z_{23} z_{52} & 0 & 0 & 1 \\
1 & 0 & 0 & 0 & 0 \\
z_{41}-z_{51} z_{43} & z_{42}-z_{52} z_{43} & 0 & 1 & 0 \\
z_{51} & z_{52} & 1 & 0 & 0
\end{array}\right)
$$

and

$$
\eta_{2}(x)=\left(\begin{array}{ccccc}
z_{11} & 1 & 0 & 0 & 0 \\
z_{21}-z_{11} z_{22} & 0 & z_{23} & z_{24} & 1 \\
1 & 0 & 0 & 0 & 0 \\
0 & 0 & z_{43} & 1 & 0 \\
0 & 0 & 1 & 0 & 0
\end{array}\right)
$$

We show how to recover the entries of $x$ as polynomials of the entries in $\eta_{1}(x)$ and $\eta_{2}(x)$. The variables $z_{51}, z_{52}, z_{43}, z_{23}, z_{24}$, and $z_{11}$ appear in $\eta_{1}(x)$ or $\eta_{2}(x)$. (These are the variables that have no 0's between them and the 1 to their right in $\eta_{1}(x)$ or $\eta_{2}(x)$.) Given these, we can recover $z_{41}, z_{42}$, and $z_{22}$ from the entries of $\eta_{1}$ and $\eta_{2}$, since $z_{41}=\left(z_{41}-z_{51} z_{43}\right)+z_{51} z_{43}, z_{42}=\left(z_{42}-z_{52} z_{43}\right)+z_{52} z_{43}$, and $z_{22}=\left(z_{22}-z_{24}\left(z_{42}-z_{52} z_{43}\right)-z_{23} z_{52}\right)+z_{23} z_{52}+z_{24}\left(z_{42}-z_{52} z_{43}\right)$. Given these, we can recover $z_{21}$, since $z_{21}=\left(z_{21}-z_{11} z_{22}\right)+z_{11} z_{22}$.

\section{Acknowledgments}

AK was supported by an NSF grant. AY was supported by an NSF grant; he also acknowledges UIUC's Center for Advanced Study, where he was a Beckman Fellow during the completion of this work.

\section{References}

[Bal12] M. Balan, Multiplicity on a Richardson variety in cominuscule $G / P$, Trans. Amer. Math. Soc., 365 (2013), 3971-3986.

[BilCos12] S. Billey and I. Coskun, Singularities of generalized Richardson varieties, Commun. Algebra, 40(4) (2012), 1466-1495.

[BilLak01] S. Billey and V. Lakshmibai, Singular loci of Schubert varieties, Progress in Mathematics 182 (2000), Birkhäuser, Boston.

[Bri02] M. Brion, Positivity in the Grothendieck group of complex flag varieties, J. Algebra 258 (2002), 137-159.

[Bri05] L_ Lectures on the geometry of flag varieties, Topics in cohomological studies of algebraic varieties, 33-85, Trends in Mathematics, Birkhäuser, Basel, 2005.

[Dar12] C. Darayon, Arithmetically Gorenstein Richardson varieties, preprint 2012.

[Har92] J. Harris, Algebraic geometry: a first course, Springer-Verlag, 1992.

[Hum75] J. Humphreys, Linear algebraic groups, Graduate Texts in Mathematics 21, Springer-Verlag, New York, 1975.

[InsYon12] E. Insko and A. Yong, Patch ideals and Peterson varieties, Transform. Groups $\mathbf{1 7}(4)$ (2012), 1011-1036.

[KazLus79] D. Kazhdan and G. Lusztig, Representations of Coxeter Groups and Hecke Algebras, Invent. Math. 53 (1979), 165-184.

[KnuLamSpe09] A. Knutson, T. Lam and D. Speyer, Projections of Richardson varieties, J. Reine Angew Math, to appear, 2012.

[KreLak04] V. Kreiman and V. Lakshmibai, Richardson Varieties in the Grassmannian, Contributions to automorphic forms, geometry, and number theory, 573-597, Johns Hopkins University Press, Baltimore, MD, 2004. 
[LiYon11] L. Li and A. Yong, Kazhdan-Lusztig polynomials and drift configurations, Algebra and Number Theory J., 5 (2011), 595-626.

[McgTra09] W. McGovern and P. Trapa, Pattern avoidance and smoothness of closures for orbits of a symmetric subgroup in the flag variety, J. Algebra, 322 (2009), 2713-2730.

[Per09] N. Perrin, The Gorenstein locus of minuscule Schubert varieties, Adv. Math., 220(2) (2009), 505-522.

[Ric92] R.W. Richardson, Intersections of double cosets in algebraic groups, Indag. Math. , N.S., 3 (1992), 69-77.

[WooYon06] A. Woo and A. Yong, When is a Schubert variety Gorenstein?, Adv. Math., 207(1) (2006), 205-220.

[WooYon08] Governing singularities of Schubert varieties, J. Algebra, 320(2) (2008), 495-520.

[Wys12] B. Wyser, Schubert calculus of Richardson varieties stable under spherical Levi subgroups, (2012), arXiv:1209.0739

[UlfWoo11] H. Úlfarsson and A. Woo, Which Schubert varieties are local complete intersections?, (2011), arXiv:1111.6146.

Department of Mathematics, Cornell University, Ithica, NY 14853-4201, USA

E-mail address: allenk@math.cornell.edu

Department of Mathematics, University of Idaho, Moscow, ID 83844-1103, USA

E-mail address: awoo@uidaho.edu

Department of Mathematics, University of Illinois at Urbana-Champaign, Urbana,

IL 61801, USA

E-mail address: ayong@illinois.edu 